\title{
Analysis of PCBs in soils and sediments by microwave-assisted extraction, headspace- SPME and high resolution gas chromatography with ion-trap tandem mass spectrometry
}

\author{
PAULO HERBERT* ${ }^{*}$, SIMONE MORAISZ, PAULA PAÍ GAz, ARMINDA \\ ALVESY and LU' CIASANTOSY
}

\begin{abstract}
A procedure for the determination of seven indicator PCBs in soils and sediments using microwave-assisted extraction (MAE) and headspace solid-phase microextraction (HS-SPME) prior to GC-MS/MS is described. Optimization of the HS-SPME was carried out for the most important parameters such as extraction time, sample volume and temperature. The adopted methodology has reduced consumption of organic solvents and analysis runtime. Under the optimized conditions, the method detection limit ranged from

0.6 to $1 \mathrm{ng} / \mathrm{g}$ when $5 \mathrm{~g}$ of sample was extracted, the precision on real samples ranged from 4 to $21 \%$ and the recovery from 69 to $104 \%$. The proposed method, which included the analysis of a certified reference material in its validation procedure, can be extended to several other PCBs and used in the monitoring of soil or sediments for the presence of PCBs.
\end{abstract}

Keywords: Microwave-assisted extraction; Solid-phase microextraction;

Polychlorinated biphenyls; Soil; Sediments

\section{Introduction}

In the last 30 years, growing awareness has been directed towards the risks posed to human health and the global environment by the increasing release of anthropogenic chemicals such as polychlorinated biphenyls (PCBs) into the environment.

There is no uniform approach to the suite of PCBs congeners most appropriate for studies of terrestrial and freshwater ecosystems. The list known as ICES7 [1] (International Council for the Exploration of the Sea: CB 28, CB 52, CB 101, CB 118, CB 138, CB 153, CB 180) has increasingly become recommended for environ- mental monitoring, because this group of PCBs contributes largely to the total amount found in most environmental samples [2].

The aim of any extraction technique in analytical chemistry is to provide effective, rapid, and quantitative removal of the analyte from its matrix, with minimal solvent usage. Several researchers have compared a range of techniques for the extraction of organic pollutants from environmental matrices [3].

Lopez-Avila et al. [4] tested microwave-assisted extraction (MAE) as an alterna- tive to Soxhlet, sonication, and SFE for the extraction of 95 organic pollutants pesticides listed in United States Environmental Protection Agency (US EPA) Method 8250. The results showed the highest recoveries for MAE and Soxhlet, but the best precision was reached with the former [4]. Moreover, the employment of MAE has many advantages over other classical 
extraction techniques, such as the reduction in extraction time and solvent consumption, and the possibility of multiple-sample analysis [5-8]. Unfortunately, MAE cannot separate the target analytes from other extractable interferences coexisting in the sample, requiring further purification steps, resulting in time-consuming and multi-step procedures. Solid-phase microextraction (SPME) coupled with high-resolution chro- matography with tandem mass spectrometry provides a solution to address this drawback.

Solid-phase microextraction has been proposed for the determination of PCBs in various matrices such as water [9-11] or soils and sediments [12]. Llompart et al. [12] extracted PCBs from soil samples by heating the slurry formed by mixing water with soil and performing headspace (HS) SPME. However, the presence of slurry in the extraction vial produces a significant decrease in the SPME efficiency. Therefore, the combination of SPME with other methods to extract the analytes in liquid or solid matrices has been studied, including MAE coupled with SPME, namely in the analysis of chlorinated pesticides in plants or water $[13,14]$, and PCBs in water or ash $[15,16]$. Furthermore, other authors reported the use of MAE as an initial exhaustive extraction step, followed by HS-SPME of the MAE extract, as a selective extraction/concentration step, thus obviating time-consuming clean-up and concentration steps [16]. To our knowledge, none reported the applica- tion of MAE combined with HS-SPME to the analysis of PCBs in sediments and/or soils. This technique provides efficient enrichment and cleanup, and also a good selectivity and sensitivity.

The aim of this study has been the modification of previously described MAE methods for PCBs by coupling HS-SPME and gas chromatography/tandem mass spec-trometry (GC-MS/MS) in order to skip the purification steps, and to analyse these compounds in soils and sediments. This work describes the development and validation of a simple and fast analytical procedure for $\mathrm{PCBs}$, including the analysis of a certified reference material (a freshwater harbour sediment), and offers further evidence of the applicability of MAE-HS-SPME-GC$\mathrm{MS} / \mathrm{MS}$ to the analysis of these compounds in solid samples. 


\section{Experimental}

\subsection{Materials}

Hexane and acetone Envisolv and ethanol analytical grade were purchased from Riedel-de Hae" $\mathrm{n}$ (Seelze, Germany). Selected indicator PCBs congeners (CB 28, CB 52, CB 101, CB 118, CB 138, $C B$ 153, and $C B$ 180) were acquired from Restek (Bellefonte, $P A$ ) as analytical standards.

The polychlorinated biphenyls (PCBs) included in this study were used to prepare a 'working standard' in ethanol with $10 \mathrm{mg} / \mathrm{L}$ of PCBs, on average. This solution was used to prepare diluted standard solutions and to spike sand samples to the requiredconcentration. To comprise $1.8 \%$ of ethanol in all diluted standard, $720 \mathrm{~mL}$ of ethanolwas added before HSSPME of each sample $(40 \mathrm{~mL})$.

Certified Reference Material-CRM 536 (freshwater harbour sediment)-was obtained from the Institute for Reference Materials and Measurements (IRMM) (Geel, Belgium) and had $12 \%$ organic matter and $1.28 \%$ moisture content (mass frac- tions). Additional information about this sediment is available on the European Commission report [17]. The certified values for the selected PCBs in this material are included in the results section. Water was distilled and deionized. Helium carrier gas (99.9999\% purity) was supplied by Praxair (Madrid).

To minimize adsorption and loss, or desorption of the studied compounds during handling and analysis, all glass material was silanized prior to utilization by soaking it overnight in a $10 \%$ dichlorodimethylsilane solution in toluene, then rinsing with toluene and methanol and drying thoroughly for $4 \mathrm{~h}$ at $400^{\circ} \mathrm{C}$.

\subsection{Soil-sample preparation}

The sandy soil sample $(\mathrm{pH} 7.8,2.18 \%$ organic matter content, $0.17 \%$ water content) used for obtaining the MAE/HS-SPME/GC/MS-MS calibration curve was air-driedand sieved to a grain size of $2 \mathrm{~mm}$. After homogenization, it was stored at $4{ }^{\circ} \mathrm{C}$, and subsequently analysed to confirm the absence of PCBs in order to be used as standard blank soil.

Spiked soil samples were prepared by adding appropriate volumes of the PCBs concentrated stock solution to a $5 \pm 0.1 \mathrm{~g}$ portion of soil. The spiked samples as well as the unknown landfill soil samples were allowed to stand for $24 \mathrm{~h}$ to air-dry and extracted by MAE thereafter.

\subsection{Microwave-assisted extraction of sediments and soil samples and SPME conditions}

Microwave-assisted extractions were performed with a MARS-X, 1500 W Microwave Accelerated Reaction System for Extraction (CEM, Mathews, NC) configured with a 14position carousel. The spiked soil samples (to obtain the MAE/HS-SPME/ GC/MS-MS calibration curve) or portions of samples were transferred quantitatively to the glass extraction vessels. Twenty millilitres of $n$-hexane-acetone (1:1) were used as extraction solvent. The operational parameters of the MARS-X apparatus were as follows: magnetron power $100 \%$; time to reach settings $10 \mathrm{~min}$; extraction tempera- ture $115^{\circ} \mathrm{C}$; extraction duration $10 \mathrm{~min}$; medium speed stirring; maximum vessel 
pressure cutoff $200 \mathrm{psi}$. After the extraction, the vessels were cooled to room tempera- ture before opening, and $15 \mathrm{~mL}$ of the supernatant was filtered through a Whatman No. 42

filter paper and evaporated to dryness under a gentle stream of nitrogen. Immediately before GC-MS analysis, the residue was redisolved with $720 \mathrm{~mL}$ of ethanol and $40 \mathrm{~mL}$ of water, and subjected to HS-SPME using the procedure described above. The SPME device (fibre and fibre holder) was purchased from Supelco (Bellefonte, PA). The fibre was coated with $100 \mathrm{~mm}$ polydimethylsiloxane (PDMS). Magnetic stir bars, PTFE-coated, $20 \times 7.5 \mathrm{~mm}$, were used in SPME. After each extraction, stir bars were rinsed consecutively with acetone, $n$-hexane, acetone and water, to prevent significant carryover between samples.

For HS-SPME, $50 \mathrm{~mL}$ (nominal size) crimp top HS vials (actual capacity about $55 \mathrm{~mL}$ ), 20 $\mathrm{mm}$ black Viton septa and aluminium seals were used, all from Supelco (Oakville, Canada). During extraction, the fibre was about $1 \mathrm{~cm}$ above the surface of the liquid and stirring set to give a vortex depth of $0.5 \mathrm{~cm}$.

Optimized HS-SPME conditions were: headspace during $60 \mathrm{~min}$ of $40 \mathrm{~mL}$ of sample $(1.8 \%$ of ethanol) at $65^{\circ} \mathrm{C}$, with $100 \mathrm{~mm}$ PDMS coated fibre.

\subsection{Chromatographic and MS/MS conditions}

For the chromatographic separation and detection of the studied compounds, a Varian (Walnut Creek, CA) CP-3800 gas chromatograph, equipped with a split/ splitless injector (model 1079), and a Varian Saturn 2000 ion trap detector were used. The analytical column was a Varian $60 \mathrm{~m} \times 0.25 \mathrm{~mm}$ CP-Sil $8 \mathrm{CB}$ lowbleed/ MS $(0.24 \mathrm{~mm}$ film thickness). Helium at $0.9 \mathrm{~mL} / \mathrm{min}$ (constant flow) was used as carriergas.

The injection temperature was $260^{\circ} \mathrm{C}$, in splitless mode. At $10 \mathrm{~min}$, the split valve was open. SPME fibres remained in the injector for at least 15 min to minimize carryover.

The oven-temperature programme was as follows: $80^{\circ} \mathrm{C}$ was held for $10 \mathrm{~min}$; then ramped to $170^{\circ} \mathrm{C}$ at $20^{\circ} \mathrm{C} / \mathrm{min}$, and to $260^{\circ} \mathrm{C}$ at $3^{\circ} \mathrm{C} / \mathrm{min}$; then to $300^{\circ} \mathrm{C}$ at $5^{\circ} \mathrm{C} / \mathrm{min}$.

After holding for $2 \mathrm{~min}$ at $300^{\circ} \mathrm{C}$, the temperature returned to initial values. The total run time was $54.5 \mathrm{~min}$.

Tandem mass spectrometry was carried out under ionization with electron impact at 70 $\mathrm{eV}$ in MS/MS mode with Multiple Reaction Monitoring (MRM).

The transfer line, manifold and trap temperatures were $290^{\circ} \mathrm{C}, 50^{\circ} \mathrm{C}$ and $210^{\circ} \mathrm{C}$, respectively. The emission current was set to $60 \mathrm{~mA}$ for all MS segments and the axial modulation voltage to $4.0 \mathrm{~V}$.

Detection was made by resonant collision-induced dissociation (CID) MS/MS, with CID frequency offset kept at zero and excitation time to $40 \mathrm{~ms}$ for all compounds. The most critical parameters were set to obtain a maximum sensitivity, and these are summarized in table 1 .

In the present work, the prescan time was set to $1500 \mathrm{~ms}$ in order to maximize the number of daughter ions formed, and a value of 2000 for the target TIC. The maximum ionization time employed was $25 \mathrm{~ms}$ for all segments.

The compounds were identified and quantified by extracting the characteristic ions of each studied compounds, monitored at the specific retention time, within a peak window of $\pm 0.2 \mathrm{~min}$. Quantification was carried out using an external standard with the most prominent ion(s) obtained in each case. 
Table 1. Ion preparation method parameters for each segment of the GC-MS/MS method. ${ }^{a}$

\begin{tabular}{|c|c|c|c|c|c|c|}
\hline Segmen & Compounds & & $\begin{array}{l}\text { Start } \\
\text { time }\end{array}$ & $\begin{array}{l}\text { End } \\
\text { time }\end{array}$ & $\begin{array}{c}\text { Precursor } \\
\text { ions }\end{array}$ & $\begin{array}{r}\text { Quantificati } \\
\text { on ions }\end{array}$ \\
\hline $\begin{array}{r}\text { Solvent } \\
\text { delay }\end{array}$ & - & & 0 & 10 & - & - \\
\hline 2 & $\begin{array}{l}2,4,4^{v} \text {-Trichlorobiphenyl } \\
2,2^{v}, 5,5^{v} \text {-Tetrachlorobiphenyl }\end{array}$ & $\begin{array}{l}\text { CB } 28 \\
\text { CB } 52\end{array}$ & 28.2 & 35.5 & $\begin{array}{l}256 \\
292\end{array}$ & $\begin{array}{l}186 \\
257\end{array}$ \\
\hline 3 & $2,2^{0}, 4,5,5^{\mathrm{U}}$-Pentachlorobiphenyl & CB 101 & 36.2 & 38.5 & 326 & 291 \\
\hline 4 & 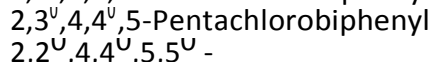 & $\begin{array}{l}\text { CB } 118 \\
\text { CB } 153\end{array}$ & 39.2 & 43 & $\begin{array}{l}326 \\
360\end{array}$ & $\begin{array}{l}256 \\
325\end{array}$ \\
\hline 5 & 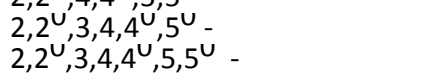 & $\begin{array}{l}\text { CB } 138 \\
\text { CB } 180\end{array}$ & 43 & 54.5 & $\begin{array}{l}360 \\
394\end{array}$ & $\begin{array}{l}325 \\
359\end{array}$ \\
\hline
\end{tabular}

${ }^{a}$ Collision-induced dissociation frequency offset was set to $0 \mathrm{~Hz}$ to all segments.

\section{Results and discussion}

Extraction is the first step in the analysis of POPs in soils, sludge, and other solid wastes. One of the most commonly used microwave-assisted solvent mixtures is hexane-acetone (1:1). Lopez-Avila et al. [4, 18, 19] found this to be advantageous for environmental contaminants such as polycyclic aromatic hydrocarbons (PAHs), organochlorine pesticides (OCPs), PCBs, phenols and organophosphorus pesticides.

The MAE procedure adopted in this study meets the requirements for sample extrac- tion of US EPA Method 3546, which recommends the use of hexane-acetone (1:1) as extractor and is suitable for the extraction of PCBs from soils, clays, sludges, solid wastes and sediments [20].

\subsection{Optimization of the HS-SPME procedure}

Solid-phase, PDMS-coated microextraction fibres, have been successfully used for selective extraction of PCBs $[9,10,12]$. Although other fibre coatings have been studied for their efficiency on extraction of some of the compounds of our interest, some even showing slightly better results than PDMS [21, 22], these are still those most used and best characterized, and were therefore used in this work.

The HS-SPME procedure was optimized by studying the effect of several param- eters on the peak area (MS operating in full scan mode) of each of the studied compounds: extraction temperature, extraction time, flask/sample volume ratio, and desorption time. These experiments were conducted on a standard solution containing, on average, $1 \mathrm{mg} / \mathrm{L}$ of the studied PCBs.

The effect of temperature on the extraction efficiency was different for each compound, depending on the volatility. Three different temperatures $\left(22,47\right.$, and $\left.65^{\circ} \mathrm{C}\right)$ were studied. For the most volatile $\mathrm{PCB}$ ( $C B$ 28), a higher extraction temperature slightly decreased the extraction efficiency.

Possibly, the rise in vapour pressure of these compounds at higher temperatures is counterbalanced by the decrease in the partition coefficient from the gas phase into the fibre. For the less volatile compounds, and thus with a greater difficulty in extracting from the headspace, increasing the extraction temperature enhances the sensitivity. To achieve an acceptable sensitivity and extraction time for all compounds, the temperature was set to $65^{\circ} \mathrm{C}$. 
As expected, the extraction efficiency increased for all the compounds with the extraction runtime. In order to maximize sample output, the extraction time was set to $60 \mathrm{~min}$ (about the same for the chromatographic separation).

As other authors reported when performing HS [15, 23], the response increases as the headspace/sample volume ratio decreases, because of the greater concentration of volatilized compounds in the gaseous fraction. Using vials with different capacities $(16,40$, and $55 \mathrm{~mL}$ ), the effect of sampling at volume ratios (total vial volume to aqueous volume) of $4,3,2.7$, and 1.4 was studied. In order to maximize the extraction efficiency while maintaining a sufficient headspace to allow the SPME fibre to stand about $1 \mathrm{~cm}$ above the liquid surface, the sample volume was set to $40 \mathrm{~mL}$ (volume ratio of 1.4) in $55 \mathrm{~mL}$ vials.

Concerning desorption time, it was observed that at least $15 \mathrm{~min}$ was needed to avoid carryover on fibre re-desorption. During analysis, each fibre stood in the injector at least $15 \mathrm{~min}$ before further use. Under the current conditions, the SPME fibre used during method validation was still presenting good adsorption capabilities after more than 60 extractions.

\subsection{Method validation}

In the present work, six standards were used for the calibration of selected PCBs (figure 1). Appropriate volumes of standard solution containing PCBs were added to $5 \mathrm{~g}$ of model soil in order to build a calibration curve from 0.51 to $5.8 \mathrm{ng} / \mathrm{g}$. Because different amounts of extracted sample could be subsequently used, the calibration curve was constructed based on the mass of each PCB present on each standard. The calibration functions were linear within the concentration range considered for each compound.

The method sensitivity decreased towards the most chlorinated (least volatile) PCBs. The best sensitivity was achieved for $\mathrm{CB} 52$ and the worst for $\mathrm{CB} 180$ (figure 1).

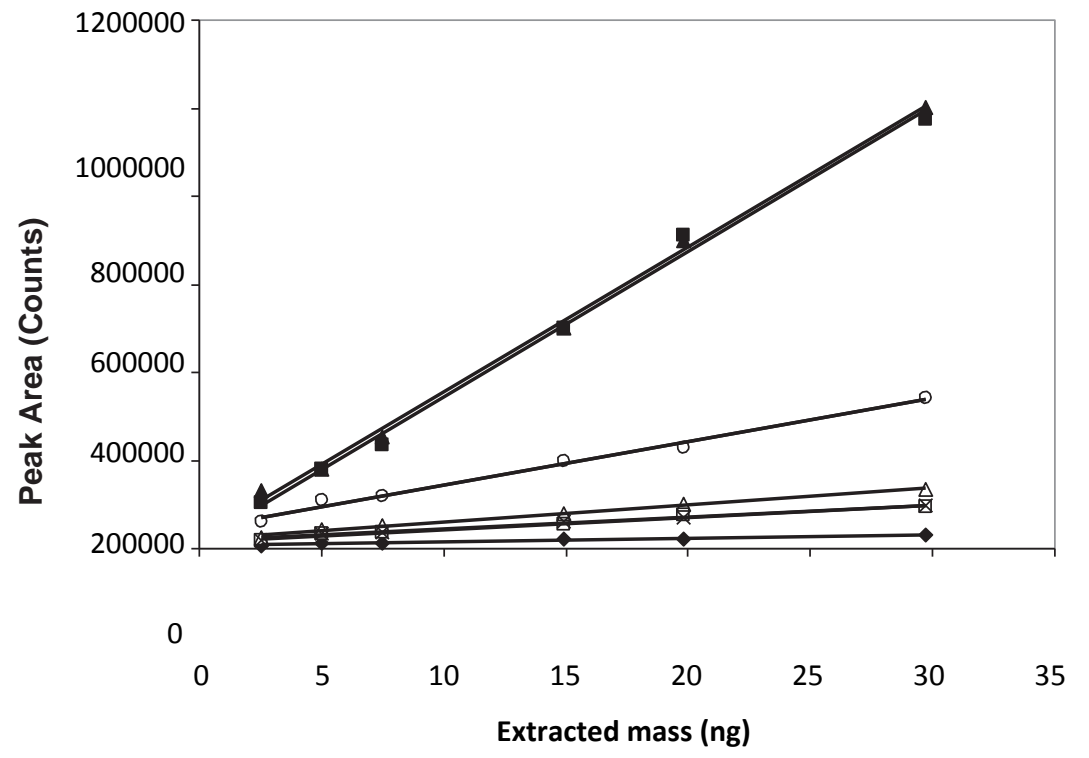

Figure 1. Standard calibration curves obtained for the selected PCBs. 
Table 2. Method validation parameters.

\begin{tabular}{lcccccc}
\hline Compoun & $\begin{array}{c}\text { Retentio } \\
\text { ntime } \\
(\mathrm{min})\end{array}$ & $\begin{array}{c}\text { Detectio } \\
\text { n } \\
\text { (ng/g) }\end{array}$ & $\begin{array}{c}\text { Quantificatib } \\
\text { on limitb } \\
(\mathrm{ng} / \mathrm{g})\end{array}$ & $\begin{array}{c}\text { Precision } \pm \\
\text { RSD\% } \\
(n 1 / 44)\end{array}$ & $\begin{array}{c}\text { Recovery (\%) } \\
\text { RSD }\left(n \frac{1}{4} 4\right)\end{array}$ & $\begin{array}{c}\text { CRM } \\
\text { certified } \\
(\mathrm{ng} / \mathrm{g})\end{array}$ \\
\hline CB 28 & 30.79 & 0.6 & 2.2 & 12 & $104 \pm 12$ & 44 \\
CB 52 & 32.46 & 0.4 & 1.2 & 21 & $74 \pm 26$ & 38 \\
CB 101 & 37.28 & 0.4 & 1.4 & 9 & $80 \pm 9$ & 44 \\
CB 118 & 40.9 & 0.7 & 2.3 & 10 & $70 \pm 11$ & 28 \\
CB 153 & 42.07 & 0.6 & 1.9 & 12 & $80 \pm 13$ & 50 \\
CB 138 & 43.66 & 0.6 & 1.9 & 4 & $69 \pm 4$ & 27 \\
CB 180 & 44.38 & 1 & 3.4 & 10 & $71 \pm 11$ & 22 \\
\hline
\end{tabular}

${ }^{\mathrm{a}}$ Calculated by $3 \times s y / x /$ calibration curve slope. ${ }^{\mathrm{b}}$ Calculated by $10 \times s y / x /$ calibration curve slope,

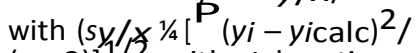

$(n-2)]^{1 / 2}$, with yi denoting experimental values and yicalc calculated by the calibration curve) and considering $5 \mathrm{~g}$ of sample extracted.

The squared correlation coefficient $\left(R^{2}\right)$ for the studied compounds ranged from 0.997 (CB 52) to 0.979 (CB 180).

Although there are other methods to calculate detection and quantification limits (based for example on the signal-to-noise ratio) with which lower detection limits values may be obtained, these parameters were assessed by the method based in the calibration curve, described by the sum of the intercept and three times $s y / x\left(s y / x^{1 / 4}\left[P^{2}(y i-y i c a l c)^{2} /(n-2)\right]^{1 / 2}\right.$, with $y i$ denoting the experimental values and yicalc calculated by the calibration curve) and are presented in table 2. Detectionlimits ranged from 0.4 (CBs 52 and 101) to 1 (CB 52) ng/g when $5 \mathrm{~g}$ of standard samples were extracted, and quantification limits were $1.2-3.4 \mathrm{ng} / \mathrm{g}$.

Microwave-assisted extraction of the certified reference material CRM 536 followed by HSSPME showed a repeatability for the whole procedure of $4-21 \%$ for all considered congeners (table 2).

In the recovery experiments, $1 \mathrm{~g}$ of the CRM 536 was extracted and $2.5 \mathrm{~mL}$ of the extract dried in order to obtain the quantification within the calibration range. However, when unknown soil samples were analysed, $5 \mathrm{~g}$ were taken, as for the calibration standards.

If compared with other methods directed to the same target analytes and sample types, the combination of MAE-HS-SPME and GC-MS/MS as studied here showed similar or better characteristics, namely in terms of quantification limits, precision, accuracy, analysis time, and solvent consumption (table 3 ).

\subsection{Analysis of real samples}

Figure 2 shows the chromatographic separation of the selected PCBs in the CP-Sil 8 analytical column obtained from a HS-SPME of MAE extracts from the CRM 536 (1 g extracted) and from a soil sample (5 g extracted) collected in a landfill ( $42.8 \%$ humidity and $2.79 \%$ total organic matter content).

The elution order obtained for PCBs compares to previously reported order for PCBs within a homologue group on non-polar stationary phases according to their number of orthochlorines [28]. The landfill soil sample was obtained from an already sealed landfill, with uncontrolled waste deposition for more than 10 years. Although 
Table 3. Comparison of the performance of some reported methods for PCBs extraction and analysis.

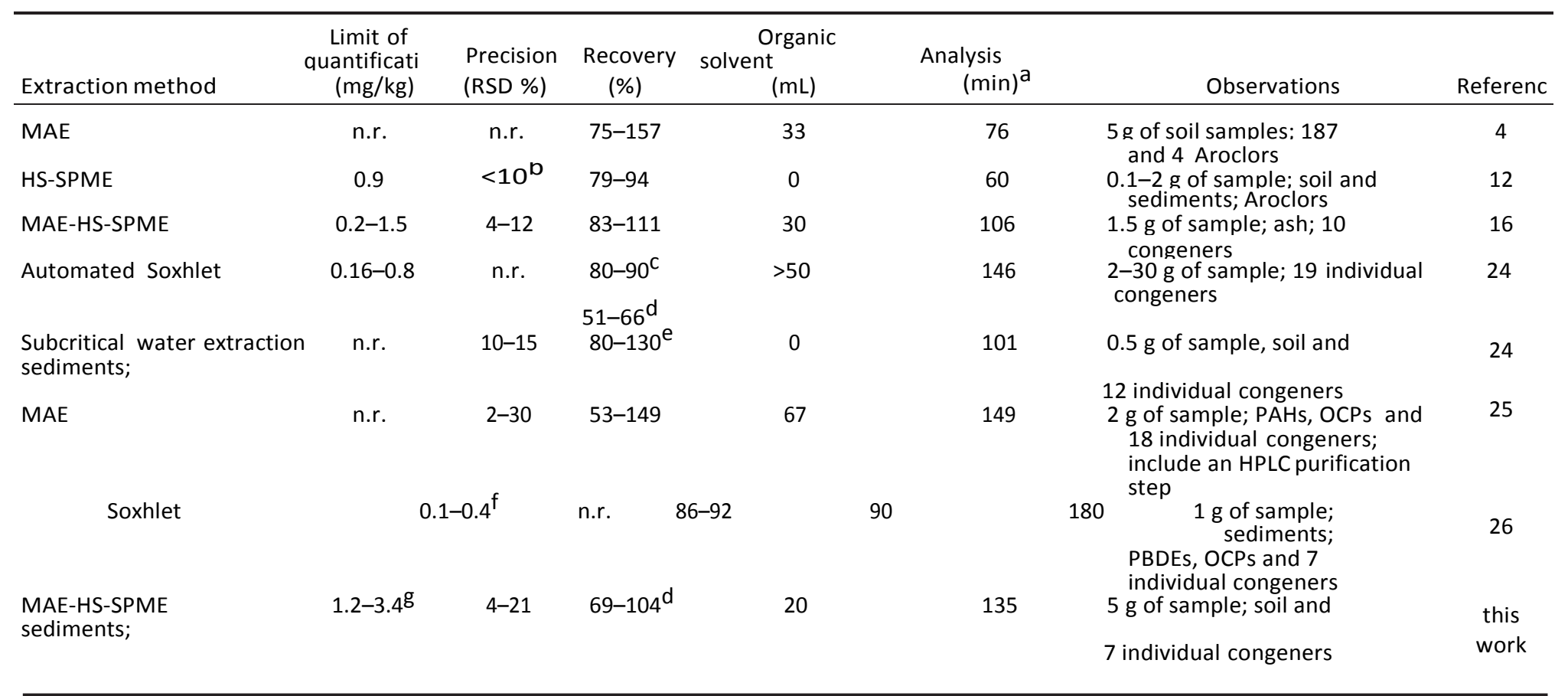

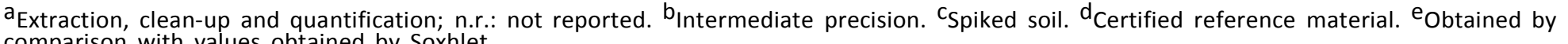
comparison with values obtained by Soxhlet.

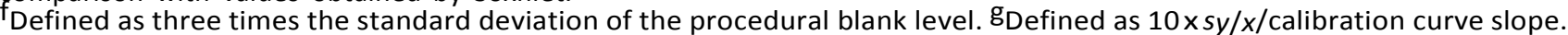





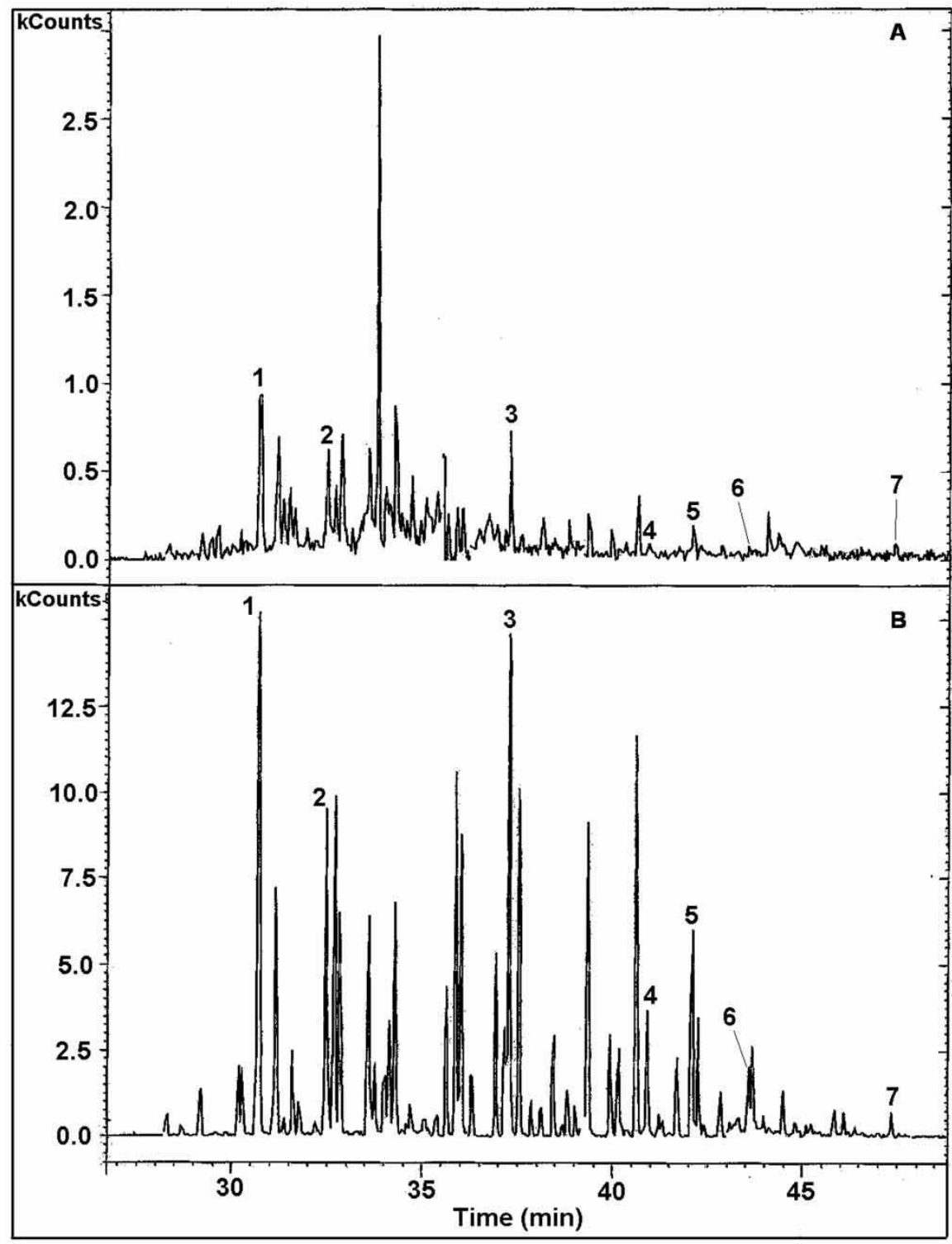

Figure 2. Extracted ion chromatograms of a landfill soil sample (A) and CRM 536 (B). (1) CB 28, (2) CB 52, (3) CB 101, (4) CB 118, (5) CB 153, (6) CB 138, (7) CB 180.

exempt of any of the studied compounds above the respective quantification limits, the analysis of the sample showed the presence of several PCBs, as confirmed by the respec- tive MS/MS spectra.

Apart from the seven indicator PCBs studied in this work, CRM 536 contains several other PCBs that can in the future be quantified by the same method and, therefore, also in the unknown soil samples or sediments, providing that these compounds are included in the standards. 


\section{Conclusions}

The combination of the microwave-assisted extraction, using a small volume of the mixture $n$-hexane-acetone $(1: 1 ; \mathrm{v} / \mathrm{v})$, followed by headspace solid-phase microextrac-tion allowed the determination of seven indicator PCBs in soil and sediments with reduced sample preparation time and very low consumption of organic solvents. The method can be further improved by including appropriate internal standard(s) in order to avoid differences between extraction efficiencies among different samples. As a part of an ongoing project, the method will be applied to a larger number of landfill soil samples and sediments.

\section{Acknowledgements}

The authors wish to thank the Fundac, ã o para a Cie^ ncia e a Tecnologia (FCT) for the grant SFRH/BPD/7155/2001 and for the financial support through the project POCTI/AGR/44491/2002 (co-financed by FEDER).

\section{References}

[1] ICES. Report of the ICES advisory Committee on marine pollution. Co-operative Research Report of the International Council for Exploration of the Sea, 167 (1986).

[2] R.T. Leah, M.S. Johnson, L. Connor, C. Levene. Mar. Environ. Res., 43, 345

(1997). [3] N. Saim, J.R. Dean, Md. P. Abdullah, Z. Zakaria. J. Chromatogr. A,

791, 361 (1997).

[4] V. Lopez-Avila, R. Young, J. Benedicto, P. Ho, R. Kim, W.F. Beckert. Anal. Chem., 67, 2096

(1995). [5] J.R. Dean, G. Xiong. Trends Anal. Chem., 19, 553 (2000).

[6] R.-A. Du"ring, St. Ga"th. Fresenius J. Anal. Chem., 368, 684 (2000).

[7] H. Budzinski, M. Letellier, S. Thompson, K. LeMenach, P. Garrigues. Fresenius J. Anal. Chem., 367, 165 (2000).

[8] N. Carro, Y. Saavedra, I. Garcia, M. Llompart. J. Microcolumn Sep., 11, 544

(1999). [9] D.W. Potter, J. Pawliszyn. Environ. Sci. Technol., 28, 298 (1994).

[10] Y. Yang, D.J. Miller, S.B. Hawthorne. J. Chromatogr. A, 800, 257 (1998).

[11] R.-A Doong, P.-L. Liao. J. Chromatogr. A., 918, 177 (2001).

[12] M. Llompart, K. Li, M. Fingas. J. Microcol. Sep., 11, 397 (1999).

[13] W.-H. Ho, S.-J. Hsieh. Anal. Chim. Acta, 428, 111 (2001).

[14] H.-P. Li, G.-C. Li, J.-F. Jen. J. Chromatogr. A, 1012, 129 (2003).

[15] Y.Y. Shu, S.S. Wang, M. Tardif, Y. Huang. J. Chromatogr. A, 1008, 1

(2003). [16] M.R. Criado, I.R. Pereiro, R.C. Torrijos. Talanta, 63, 533 (2004).

[17] J.W.M. Wegener, E.A. Maier, G.N. Kramer, W.P. Cofino (Eds). Report Eur 17799EN, p. 59, Office for Official Publications of the European Communities, Luxembourg (1997).

[18] V. Lopez-Avila, R. Young, W.F. Beckert. J. AOAC Int., 81, 462 (1998).

[19] V. Lopez-Avila, J. Benedicto. J. AOAC Int., 81, 1224 (1998).

[20] Environmental Protection Agency (Ed.). EPA Method 3546: Microwave extraction of VOC's and SVOCS (organophosphorus pesticides, organochlorine pesticides, chlorinated herbicides, phenoxy acid herbicides, PCBs). US Environmental Protection Agency, Cincinnati, $\mathrm{OH}$ (2000).

[21] A.L. Lopes, F. Augusto. J. Chromatogr. A, 1056, 13 (2004).

[22] M.R. Criado, I.R. Pereiro, R.C. Torrijos. J. Chromatogr. A, 1056, 263 (2004).

[23] B.D. Page, G. Lacroix. J. Chromatogr. A, 757, 173 (1997).

[24] Environmental Protection Agency (Ed.). In Test Methods for Evaluating Solid Waste, Physical/Chemical Methods EPA SW-846 [Version 2 (December 1997), Integrated Manual through Update III] Washington, DC (1997).

[25] S.B. Hawthorne, C.B. Grabanski, K.J. Hageman, D.J. Miller. J. Chromatogr. A, 814, 151 (1998).

[26] S. Thompson, H. Budzinski, K. LeMenach, M. Letellier, P. Garrigues. Anal. Bioanal. Chem., 372, 196 (2002).

[27] A. Covaci, A. Gheorghe, S. Voorspoels, J. Maervoet, E.S. Redeker, R. Blust, P. Schepens.

Environ. Int., 31, 367 (2005).

[28] J.W. Cochran, G.M. Frame. J. Chromatogr. A, 843, 323 (1999). 